\title{
ANNA LUSHENKOVA FOSCOLO, Les Artistes-lecteurs chez Marcel Proust et Ivan Bounine
}

\section{Roberta Sapino}

\section{(2) OpenEdition \\ 1 Journals}

\section{Edizione digitale}

URL: https://journals.openedition.org/studifrancesi/14329

DOI: $10.4000 /$ studifrancesi. 14329

ISSN: 2421-5856

\section{Editore}

Rosenberg \& Sellier

\section{Edizione cartacea}

Data di pubblicazione: 1 août 2018

Paginazione: 382-383

ISSN: 0039-2944

\section{Notizia bibliografica digitale}

Roberta Sapino, «Anna Lushenkova foscolo, Les Artistes-lecteurs chez Marcel Proust et Ivan Bounine», Studi Francesi [Online], 185 (LXII | II) | 2018, online dal 01 août 2018, consultato il 15 novembre 2021. URL: http://journals.openedition.org/studifrancesi/14329; DOI: https://doi.org/10.4000/studifrancesi. 14329

Questo documento è stato generato automaticamente il 15 novembre 2021.

\section{(c) (†)}

Studi Francesi è distribuita con Licenza Creative Commons Attribuzione - Non commerciale - Non opere derivate 4.0 Internazionale. 


\title{
ANNA LUSHENKOVA FOSCOLO, LeS Artistes-lecteurs chez Marcel Proust et Ivan Bounine
}

\author{
Roberta Sapino
}

\section{NOTIZIA}

ANNA LUSHENKOVA foscolo, Les Artistes-lecteurs chez Marcel Proust et Ivan Bounine, Paris, Classiques Garnier, 2017, 414 pp.

Due opere la cui portata di innovazione ha superato di gran lunga i rispettivi confini nazionali sono chiamate a dialogare nel denso volume di Anna LUSHENKOVA Foscolo: À la recherche du temps perdu di Marcel Proust e Žizn' Arsen'eva Ûnost' di Ivan Bunin. Nell'addentrarsi in due opere che, indipendentemente l'una dall'altra, hanno rivoluzionato il genere del romanzo autobiografico (anche se, suggerisce l'autrice, Bunin potrebbe aver letto Proust prima di quanto abbia mai ammesso di averlo fatto), la studiosa si propone di portare alla luce il ruolo cruciale che la lettura gioca da un lato nella scoperta della vocazione autoriale, dall'altro nel processo di scrittura. Il risultato è una densa indagine che si dipana lungo tre assi di ricerca, nelle quali la lettura è concepita rispettivamente come esperienza, come processo e come componente fondamentale dell'atto creativo («Introduction», pp. 9-35).

2 Nella prima parte, intitolata «Lectures vitales, lectures dangereuses» (pp. 39-130), si osserva che l'atteggiamento dello scrittore fa della lettura una vera e propria esperienza in senso fenomenologico (Lectures pour la vie, pp. 39-66): in quanto momento di "immagazzinamento di impressioni", la lettura offre all'autore la materia a partire dalla quale creare la propria opera, non come un modello da copiare, ma come una tavolozza della quale servirsi liberamente. Così concepita, la lettura avviene nella sintesi di tre spazi: quello testuale del libro, lo spazio interiore del lettore, e il contesto immediato nel quale avviene l'atto di lettura. Il testo e lo spazio extra-testuale 
contribuiscono allora in ugual modo all'elaborazione delle "immagini mentali" (dipendenti non solo dalla sensibilità e dall'intelletto, ma anche dalla sfera affettiva) e, nutrendo il "corpo vitale" del lettore-artista, sollecitano la capacità di comprendere il proprio "libro interiore".

3 L'atteggiamento nei confronti della lettura, afferma Lushenkova Foscolo, costituisce tanto per Proust quanto per Bunin una lente attraverso la quale caratterizzare alcuni personaggi. Le Lectures mortiferes (pp. 67-130) delle quali si fa menzione non sono allora libri pericolosi per il loro contenuto, ma atteggiamenti in grado di annullare i poteri vivificanti della lettura perché fondati sulla percezione del libro come semplice oggetto. Per esempio: la bibliofilia, la convenzionalità delle letture interne ai circoli mondani o politici, la tentazione di giudicare il libro in base alle idee politiche dell'autore o alla sua abilità nella conversazione, la ricerca di una funzione didattica nei testi.

4 Intitolata «L'artiste-lecteur, initiations et incitations» (pp. 131-251), la seconda parte ripercorre l'iniziazione alla lettura (e, conseguentemente, alla scrittura) dei protagonisti dei testi che compongono il corpus. Sia nella Recherche sia in Žizn' Arsen'eva Unost' il primo incontro con la lettura avviene per mezzo non della parola scritta, ma delle immagini, rispettivamente di una lanterna magica e di un volume illustrato del Don Quichotte. Pur passando attraverso una forma percettiva-la vistaprevalentemente passiva, quest'esperienza si configura come attiva in virtù della sua capacità di sollecitare l'introspezione e aprire l'accesso a un mondo immaginario. L'iniziazione ha luogo ugualmente attraverso l'ascolto delle letture ad alta voce proposte dalla prozia e dalla mamma (in Proust) e dal precettore (in Bunin): la gioia e l'emozione contribuiscono alla creazione di legami semantici tra le parole inscindibili dalla componente affettiva dell'esperienza (Les «émois joyeux» des «charmantes lectures de l'enfance», pp. 133-157).

5 In seguito (L'éveil par les «lectures magiques», pp. 159-251), Anna Lushenkova Foscolo si sofferma sugli elementi che, in modi e momenti diversi, permettono di comparare la lettura al sonno. Svolgendosi prevalentemente di notte, per lo più quando il protagonista è in posizione orizzontale e isolato rispetto alla società, e stimolando uno stato di sonno-veglia simile a quello provocato dall'ipnosi, la lettura - come anche il sogno - presenta agli occhi del personaggio le immagini che provengono dal suo stesso mondo interiore. Allo stesso modo, la scrittura trova nel silenzio notturno il suo spazio privilegiato, mostrando il suo legame intrinseco non solo con la lettura, il sonno e il sogno, ma anche con le nozioni di morte e passione. L'attività del lettore, così come è vissuta dai due protagonisti, non è dunque priva di pericoli: l'ansia di perdere il proprio "io profondo" tra le maglie delle parole altrui è uno dei punti problematici dei romanzi analizzati, e fa della lettura un'esperienza non solo piacevole, ma anche angosciosa, talvolta non lontana dall'alienazione o dalla follia. Pur senza sottovalutare gli aspetti estetici, per entrambi i personaggi il valore intrinseco di un'opera letteraria deriva allora soprattutto dal giudizio soggettivo rispetto alla sua capacità di sollecitare l'interiorità del lettore e di portare quest'ultimo "sulla via dell'arte".

«Lire en artiste, écrire en artiste» è il titolo della terza e ultima parte (pp. 253-358), nella quale si analizza l'evoluzione del rapporto dell'artista-autore con la lettura, a cominciare dalle nozioni di Appropriation et dépassement (pp.257-316). Oggetto dell'inchiesta è innanzitutto la postura necessaria perché l'atto di lettura si trasformi in atto creativo: per entrambi gli autori, la condizione fondamentale è che la lettura abbia 
luogo al di fuori di qualsiasi automatismo, perché solo così l"'io profondo" del lettore può essere interpellato. In particolare, Proust e Bunin elaborano definizioni analoghe dell'atteggiamento dell'artista-lettore, riassumibili con la formula proustiana di "interesse disinteressato", che consiste in una volontà di appropriazione non egoistica, bensì legata al desiderio di condividere. In questa prospettiva, l'attenzione del lettore dovrà essere non selettiva, ma "dispersa", al fine di percepire la pluralità (prossima alla polifonia bachtiniana) dell'opera. Tale postura è produttiva non solo in rapporto alla parola scritta, ma anche per quanto riguarda La lecture et l'écriture de la vie (pp. 317-358). La lettura, infatti, da un lato permette all'artista di esperire la sintesi tra avvenimenti lontani nel tempo, dall'altro favorisce l'emancipazione dello sguardo necessaria alla creazione. Come leggono i libri, i lettori-artisti creati da Proust e Bunin decifrano gli individui, i luoghi, i fenomeni che li coinvolgono.

7 In conclusione ("Conclusion», pp. 359-362), questa ricca monografia dimostra bene come la lettura possa costituire una chiave particolarmente interessante per comprendere la concezione di un artista non solo della propria scrittura o dell'arte in generale, ma anche delle relazioni tra l'arte e la realtà. Il metodo utilizzato costituisce allora un modello potenzialmente valido per lo studio di altri romanzi di formazione artistica. 